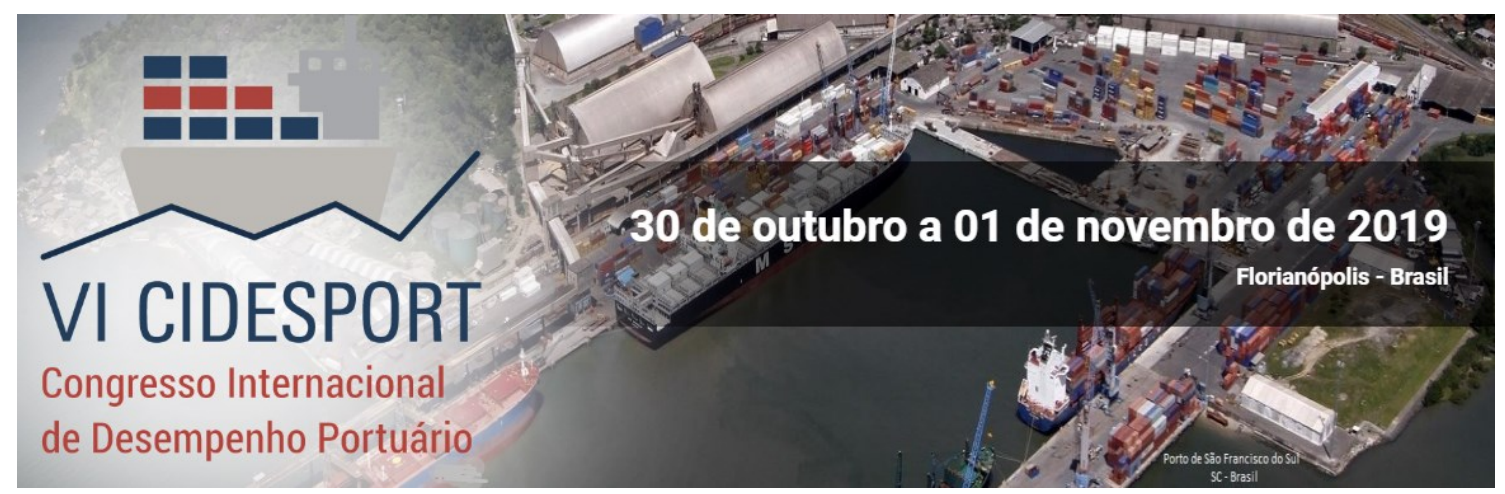

\title{
O IMPACTO DA CABOTAGEM NA GESTÃO DOS PORTOS: UMA REVISÃO SISTEMÁTICA DE LITERATURA
}

\author{
Thauane Adamoli Amaral \\ Universidade Federal do Rio Grande \\ Kauê Costa Rego da Silva \\ Universidade Federal do Rio Grande
}

Ticiani Chaves Gonçalves

Universidade Federal do Rio Grande

Diogo Garcia Storino

Universidade Federal do Rio Grande

André Andrade Longaray

Universidade Federal do Rio Grande

\begin{abstract}
Resumo: O Brasil possui uma longa extensão de costa, o que propicia o uso de transportes por via aquática um meio de criar um maior desenvolvimento econômico para o país. Um dos modais mais utilizados e eficientes é o de cabotagem. Nesse sentido, o presente estudo tem como objetivo realizar uma investigação da literatura sobre o modal de transporte cabotagem e seus impactos na gestão portuária no cenário mundial. No que tange a metodologia de pesquisa, é definida a partir do propósito, o qual se classifica como pesquisa-diagnóstico. Quanto ao caráter o estudo enquadra-se como quantitativo. $O$ delineamento da pesquisa é descritivo. A coleta de dados foi a partir de bases de dados online, com inserção de 27 combinações de palavra-chave em pares em cada base de dados. Inicialmente, selecionou-se as bases de dados alinhadas a área de pesquisa, totalizando em quatro bases de dados, IEEE, Science Direct, Scopus e Web of Science. Posteriormente, realizou-se a análise bibliométrica acerca da produção e autoria, conteúdo, citação dos artigos e estudo das referências bibliográficas dos artigos pertencentes ao portfólio.
\end{abstract}

Palavras-chave: Cabotagem. SSS. Análise bibliométrica.

\section{INTRODUÇÃO}

O Brasil possui uma longa extensão de costa, o que propicia o uso de transportes por via aquática um meio de criar um maior desenvolvimento econômico para o país. Um dos modais mais utilizados e eficientes é o de cabotagem, que é definido como a navegação realizada entre portos do país pelo litoral ou por vias fluviais, se contrapondo à navegação de longo curso, ou seja, aquela realizada entre portos de diferentes países, envolvendo toda uma cadeia logística de transporte de cargas (FLORENTINO, 2010; MOURA; BOTTER, 2011).

\footnotetext{
* A revisão gramatical, ortográfica, ABNT ou APA foi realizada pelos autores.
} 


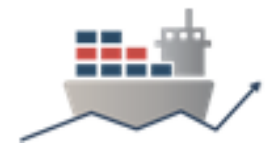

A cabotagem está ligada ao conceito de multimodalidade, aliada aos demais modais de transportes, ferroviário, rodoviário e hidroviário, pode contribuir muito para redução dos custos logísticos nacionais, além de reduzir avarias de transporte e perda de carga (MOURA; BOTTER, 2011; BENDALL; BROOKS, 2010).

De acordo com a Agência Nacional de Transporte Aquaviários - ANTAQ (2019b), a navegação de longo curso brasileira transportou 822,8 milhões de toneladas. O número representa um crescimento de 2,25\% em relação a 2017 , quando foram transportadas 804,6 milhões de toneladas.

Conforme a ANTAQ (2019a), o estudo desenvolvido pela Associação Brasileira dos Armadores de Cabotagem (ABAC), demonstra que a navegação de cabotagem emite quatro vezes menos poluentes que o transporte rodoviário. Há, ainda, um potencial de economia de $\mathrm{R} \$ 1,7$ bilhão por ano no frete. Além disso, poderia haver redução de dez mil acidentes nas estradas brasileiras ao ano a partir do efetivo uso da cabotagem. Para cada contêiner na cabotagem, há 9,7 contêineres potenciais nas rodovias. É destacado que no período entre os anos de 2018 e 2022, a meta é captar 4,8 contêineres entre os potenciais que estão nas rodovias.

O transporte na navegação de cabotagem cresceu $2,98 \%$ em 2018. Foram 161,2 milhões de toneladas. Em 2017, esse número alcançou 156,6 milhões de toneladas. A carga que a navegação de cabotagem mais transportou em 2018 foi de combustíveis minerais e óleos minerais, com 119 milhões de toneladas. Em seguida, minérios, e depois contêineres (ANTAQ, 2019b).

Segundo a ANTAQ (2019b), a movimentação em vias interiores foi de 98,7 milhões de toneladas no ano de 2018 , queda de $3 \%$ em relação a 2017 . No longo curso em vias interiores, foram movimentados 40,9 milhões de toneladas no ano de 2018. Na navegação interior, este número foi 37,3 milhões de toneladas. A cabotagem em vias interiores registrou 20,4 milhões de toneladas.

Frente ao exposto, o presente estudo tem como objetivo geral caracterizar a produção científica referente à temática do impacto do modal cabotagem na gestão dos portos. Os objetivos específicos são: identificar qual a relevância no cenário mundial, a partir da busca de publicações nas bases de dados: IEEE, Scopus, Web of Science e Science Direct, sobre o tema de pesquisa; analisar, a partir da análise bibliométrica, quanto ao estudo de produção e autoria, análise de conteúdo, de citação e das referências bibliográficas dos artigos.

Tendo em vista a disposição do presente trabalho, após o marco introdutório, na seção 2, é apresentado o referencial teórico acerca do tema de pesquisa. Na seção 3 , está representado a metodologia utilizada para análise do estudo. Após, na seção 4 , estão demonstrados os resultados de pesquisa. Por fim, na seção 5 , é apresentado as considerações finais, bem como, as lacunas e sugestões para trabalhos futuros.

\section{REFERENCIAL TEÓRICO}

Existem muitas definições diferentes de cabotagem marítima. No nível internacional, não existe uma definição legal única de cabotagem que seja vinculante para todos os Estados membros das Nações Unidas. No entanto, existem muitas definições regionais e nacionais de cabotagem que podem ser aplicadas. A palavra cabotagem pode ser utilizada em alguns países como "coastal trade", "coasting trade", "costwise trade" ou "domestic trade" (SEAFERS' RIGHTS INTERNACIONAL - SRI, 2018).

De acordo com a Lei $n^{\circ}$ 9.432/97 de 8 de janeiro de 1997 que dispõe sobre a ordenação do transporte aquaviário e dá outras providências, define se navegação de 
cabotagem como "a realizada entre portos ou pontos do território brasileiro, utilizando a via marítima ou esta e as vias navegáveis interiores". É denominado, também, como transporte marítimo realizado entre dois portos da costa de um mesmo país, se a navegação ocorrer entre um porto costeiro e um fluvial chama-se cabotagem, caso ocorra entre dois portos fluviais não é considerada cabotagem e sim navegação interior (BRASIL, 1997; FLORENTINO, 2010).

Contudo, tem-se também o emprego do termo Short Sea Shipping (SSS), que ao traduzi-lo é definido como transporte marítimo de curta distância. A European Commissian sugeriu em 1999 uma definição funcional para SSS, o qual é definido como a circulação de mercadorias e passageiros por mar entre portos situados na Europa geográfica ou entre esses portos e portos situados em países não europeus que tenham uma linha costeira nos mares confinados que fazem fronteira com a Europa. O SSS inclui o transporte marítimo doméstico e internacional, incluindo os serviços de alimentação - para que o frete (geralmente contêineres) seja consolidado ou redistribuído para um serviço de alto mar em um desses portos -, ao longo da costa e para as ilhas, rios e lagos (EUROPEAN COMMISSIAN, 1999).

A cabotagem ou SSS pode ser considerado um modo de transporte mais respeitador do ambiente, em particular devido aos seus custos externos comparativamente baixos e à elevada eficiência energética. Em relação as vantagens da cabotagem sobre o modal rodoviário, é possível citar emissão de $\mathrm{CO}_{2}$. O transporte marítimo em geral apresenta uma taxa menor de emissão se comparado a outros modais, como demonstrada na Figura 1, o qual mostra as médias da União Europeia (EUROPEAN COMMISSION, 1999; IFEU, 2008, IFEU, 2014, IMO, 2014 apud BARBOSA, 2018).

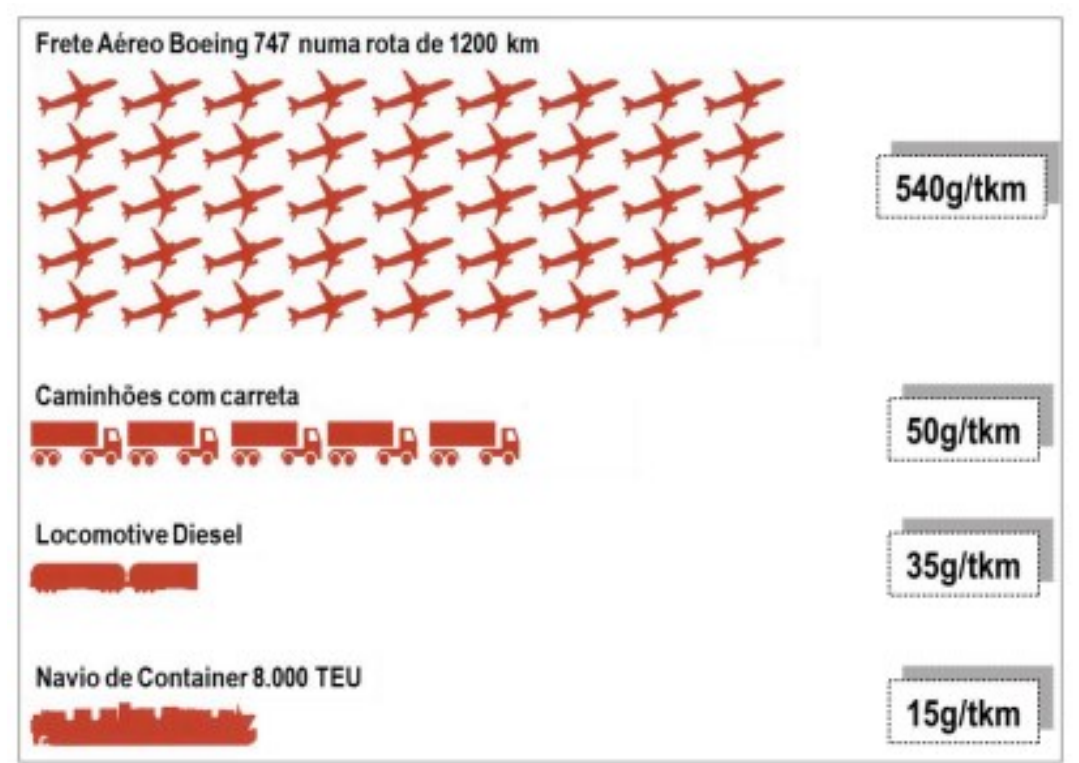

Figura 1 - Taxas de emissão de $\mathrm{CO}_{2}$ médios em g/t.km, calculado com base no mix de energia da lista de veículos padrão da comunidade europeia (EU 25)

Fonte: IFEU, 2008, IFEU, 2014, IMO, 2014 apud Barbosa, 2018.

Logo, o transporte marítimo apresenta uma diminuição de $70 \%$ na taxa de emissão de $\mathrm{CO}_{2}$ considerando o mesmo volume de carga transportada. Outro fator importante é o preço de operação de algumas operações, como a do arroz do sul catarinense para o nordeste, que pode ser reduzido em 30\% (SILVA, 2014) se comparado ao transporte rodoviário. É possível citar a maior segurança do transporte aquaviário se comparado ao rodoviário. 
Os fatores ambientais, custo e a segurança do trajeto auxiliam no desenvolvimento da cabotagem no Brasil. A partir dos anuários estatísticos aquaviários de 2010 a 2018 da ANTAQ, pode-se analisar o aumento do volume de carga movimentada por esse modal, onde houve o crescimento de toneladas movimentadas de 2010 em comparação ao ano de 2018 (Figura 2).



Figura 2 - Movimentação de cargas por toneladas via cabotagem nos anos de 2010 a 2018 Fonte: Anuário estatístico aquaviário (ANTAQ, 2010; ANTAQ, 2011, ANTAQ, 2012; ANTAQ, 2013; ANTAQ, 2014; ANTAQ, 2015; ANTAQ, 2016; ANTAQ, 2017; ANTAQ, 2018).

De acordo com o relatório executivo do Plano Nacional de Logística - PNL (2015), os deslocamentos de cargas no Brasil produziram um total de 2.386,7 bilhões de toneladas por quilometro útil (TKU) no ano de 2015. Destaca-se a elevada participação do transporte rodoviário, com $65 \%$ do total; a moderada utilização do transporte aquaviário (cabotagem marítima e hidrovias interiores), com 16\%; o transporte ferroviário, com 15\%; a participação limitada do transporte dutoviário, com $4 \%$; e a movimentação do transporte aeroviário, com percentual próximo de zero.

\section{METODOLOGIA}

No que tange a metodologia de pesquisa, é definida a partir do propósito do projeto, do caráter, do delineamento da pesquisa, das técnicas de coleta e a análise de dados a ser utilizada (ROESCH, 2015). Quanto ao propósito, é classificada como pesquisa-diagnóstico, onde almejam explorar o ambiente organizacional e de mercado, levantar e definir problemas (ROESCH, 2015).

O presente trabalho possui caráter quantitativo, pois reúne buscas em bases de pesquisas onde os artigos selecionados são transformados em dados estatísticos para análise.

Quanto ao delineamento, que visa expor os procedimentos metodológicos e técnicos de coleta e análise de dados, a pesquisa enquadra-se como descritiva, onde tem o objetivo de descrever as características de determinada população, ou o estabelecimento de relações entre variáveis (GIL, 2010). Assim, a população de pesquisa é composta pelos artigos do portifólio.

A técnica de coleta de dados, fez-se a partir da análise bibliométrica que, de acordo com Gil (2010), consiste em um estudo sistematizado utilizando como base 
materiais publicados em livros, revistas, jornais, redes eletrônicas e outros materiais acessíveis para posteriormente realizar uma apresentação dos dados.

\section{ANÁLISE BIBLIOMÉTRICA}

A análise bibliométrica tem como objetivo realizar a quantificação da produção científica por meio da análise estatística. Neste sentido, na bibliometria ou análise bibliométrica, os dados quantitativos são contabilizados a partir das publicações ou elementos que reúnem uma série de técnicas estatísticas (SILVA; HAYASHI; HAYASHI, 2011).

À vista disso, o presente estudo, será divido em duas etapas. A primeira etapa apresenta a coleta dos dados a partir das bases de dados: IEEE, Science Direct, Scopus e Web of Science. E por fim, a segunda etapa constitui na análise dos dados por meio da análise bibliométrica.

\subsection{Coleta de dados}

No que tange a coleta de dados, fez-se a partir de bases de dados online no mês de julho de 2019, por meio de combinações de palavra-chave em pares. Inicialmente, selecionou-se as bases de dados alinhadas a área de pesquisa, totalizando em quatro bases de dados, IEEE, Science Direct, Scopus e Web of Science. À vista disso, definiu-se 27 combinações de palavras-chave alinhadas ao tema de pesquisa. Combinou-se três palavras, Impact, Management e Investment, com nove palavras, cabotage, short sea shipping, internal navigation, internal shipping, domestic shipping, costal shipping, transport cost, cabotage logistics e waterway transportation network.

Como marco inicial da pesquisa, inseriu-se as combinações de palavras-chave em pares nas bases de dados, onde obteve-se 7.943 publicações. Após realizou-se a importação das publicações para um software bibliográfico, totalizando em 7.774 documentos. Na Tabela 1 está disposto os resultados, publicações e respectiva base de dados por combinação de palavra-chave, após a importação para o software.

Tabela 1 - Dados da pesquisa nas bases de dados após importação

\begin{tabular}{c|c|c|c|c|c|c}
\hline & Eixos & \multicolumn{5}{c}{ Bases } \\
\hline $\begin{array}{c}\text { Impacto I } \\
\begin{array}{c}\text { Administração } \\
\text { Portuária }\end{array}\end{array}$ & Cabotagem & $\begin{array}{c}\text { Web of } \\
\text { Science }\end{array}$ & Scopus & $\begin{array}{c}\text { Science } \\
\text { Direct }\end{array}$ & IEEE & Total \\
\hline Impact & Cabotagem & 10 & 20 & 3 & 0 & $\mathbf{3 3}$ \\
\hline & Short sea shipping & 188 & 134 & 18 & 15 & $\mathbf{3 5 5}$ \\
\hline & Internal navigation & 104 & 185 & 19 & 26 & $\mathbf{3 3 4}$ \\
\hline & Internal shipping & 212 & 85 & 22 & 22 & $\mathbf{3 4 1}$ \\
\hline & Domestic shipping & 124 & 139 & 25 & 1 & $\mathbf{2 8 9}$ \\
\hline & Coastal shipping & 796 & 518 & 113 & 75 & $\mathbf{1 . 5 0 2}$ \\
\hline & "Transport cost" & 125 & 619 & 56 & 3 & $\mathbf{8 0 3}$ \\
\hline & Cabotage logistics & 2 & 5 & 1 & 0 & $\mathbf{8}$ \\
\hline & Waterway transportation & 30 & 107 & 6 & 5 & $\mathbf{1 4 8}$ \\
\hline & network & 6 & 9 & 0 & 0 & $\mathbf{1 5}$ \\
\hline & Cabotagem & 81 & 82 & 11 & 15 & $\mathbf{1 8 9}$ \\
\hline & Short sea shipping & 150 & 293 & 25 & 84 & $\mathbf{5 5 2}$ \\
\hline & Internal navigation & 0 & 108 & 17 & 42 & $\mathbf{1 6 7}$ \\
\hline & Internal shipping & 119 & 112 & 13 & 18 & $\mathbf{2 6 2}$ \\
\hline & Domestic shipping & 494 & 461 & 77 & 104 & $\mathbf{1 . 1 3 6}$ \\
\hline & Coastal shipping & & & & & 199 \\
\hline
\end{tabular}




\begin{tabular}{|c|c|c|c|c|c|c|}
\hline & "Transport cost" & 62 & 467 & 16 & 35 & $\mathbf{5 8 0}$ \\
\hline & Cabotage logistics & 1 & 0 & 0 & 0 & $\mathbf{1}$ \\
\hline $\begin{array}{c}\text { Waterway transportation } \\
\text { network }\end{array}$ & 31 & 155 & 7 & 22 & $\mathbf{2 1 5}$ \\
\hline Investment & Cabotagem & 4 & 10 & 0 & 0 & $\mathbf{1 4}$ \\
\hline & Short sea shipping & 30 & 44 & 9 & 2 & $\mathbf{8 5}$ \\
\hline & Internal navigation & 7 & 20 & 2 & 3 & $\mathbf{3 2}$ \\
\hline & Internal shipping & 26 & 30 & 7 & 4 & $\mathbf{6 7}$ \\
\hline & Domestic shipping & 27 & 50 & 11 & 1 & $\mathbf{8 9}$ \\
\hline & Coastal shipping & 33 & 46 & 9 & 7 & $\mathbf{9 5}$ \\
\hline & "Transport cost" & 60 & 306 & 26 & 5 & $\mathbf{3 9 7}$ \\
\hline & Cabotage logistics & 0 & 2 & 0 & 0 & $\mathbf{2}$ \\
\hline & Waterway transportation & 17 & 41 & 4 & 1 & $\mathbf{6 3}$ \\
\hline & network & $\mathbf{2 . 7 3 9}$ & $\mathbf{4 . 0 4 8}$ & $\mathbf{4 9 7}$ & $\mathbf{4 9 0}$ & $\mathbf{7 . 7 7 4}$ \\
\hline
\end{tabular}

Fonte: Dados de pesquisa.

Via software bibliográfico, realizou-se a filtragem dos documentos. Incialmente excluiu-se as publicações duplicadas, onde excluiu-se 2.880 publicações duplicadas, sendo assim, reduziu-se o número de documentos para 4.894 .

Ao realizar o filtro de tipo de documento, priorizou-se artigos publicados em jornais ou revistas online como principal documento de análise. Neste sentido, ao aplicar o filtro, excluiu-se 1.634 publicações não alinhadas ao filtro, restando 3.260 documentos.

Após aplicou-se o filtro pela área da revista/jornal cientifico não alinhados á temática, resultando em 1.821 artigos.

O próximo filtro consistiu na leitura do título, resumo e texto completo dos artigos. À vista disso, fez-se a leitura dos títulos onde exclui-se as publicações onde o título não se enquadrava ao tema de pesquisa. restando 77 artigos com o título alinhado à pesquisa. Após realizou-se a leitura do resumo das publicações, resultado em 15 artigos. Por fim, filtrou-se os documentos pela leitura completa, contudo, um artigo não estava disponível gratuitamente. Após a leitura integral dos artigos obtevese oito documentos alinhados ao tema, os quais estão dispostos no Apêndice A.

$\mathrm{Na}$ Tabela 2 está representado a disposição dos resultados de artigos por combinações de palavras-chave e bases de dados.

Tabela 2 - Filtragem das publicações alinhados à pesquisa

\begin{tabular}{|c|c|c|c|c|c|c|}
\hline \multicolumn{2}{|c|}{ Eixos } & \multicolumn{5}{|c|}{ Bases } \\
\hline $\begin{array}{c}\text { Impacto I } \\
\text { Administração } \\
\text { Portuária }\end{array}$ & Cabotagem & $\begin{array}{l}\text { Web of } \\
\text { Science }\end{array}$ & Scopus & $\begin{array}{c}\text { Science } \\
\text { Direct }\end{array}$ & IEEE & Total \\
\hline \multirow[t]{9}{*}{ Impact } & Cabotage & 0 & 1 & 0 & 0 & 1 \\
\hline & Short sea shipping & 1 & 0 & 0 & 0 & 1 \\
\hline & Internal navigation & 0 & 0 & 0 & 0 & 0 \\
\hline & Internal shipping & 0 & 0 & 0 & 0 & 0 \\
\hline & Domestic shipping & 0 & 0 & 0 & 0 & 0 \\
\hline & Coastal shipping & 0 & 1 & 0 & 0 & 1 \\
\hline & "Transport cost" & 0 & 0 & 0 & 0 & 0 \\
\hline & Cabotage logistics & 0 & 0 & 0 & 0 & 0 \\
\hline & $\begin{array}{c}\text { Waterway } \\
\text { transportation network }\end{array}$ & 0 & 0 & 0 & 0 & 0 \\
\hline \multirow[t]{4}{*}{ Management } & Cabotagem & 0 & 0 & 0 & 0 & 0 \\
\hline & Short sea shipping & 1 & 1 & 0 & 0 & 2 \\
\hline & Internal navigation & 0 & 0 & 0 & 0 & 0 \\
\hline & Internal shipping & 0 & 0 & 0 & 0 & 0 \\
\hline
\end{tabular}




\begin{tabular}{|c|c|c|c|c|c|c|}
\hline & Domestic shipping & 0 & 1 & 0 & 0 & 1 \\
\hline & Coastal shipping & 1 & 0 & 0 & 0 & 1 \\
\hline & "Transport cost" & 0 & 0 & 0 & 0 & 0 \\
\hline & Cabotage logistics & 0 & 0 & 0 & 0 & 0 \\
\hline & $\begin{array}{c}\text { Waterway } \\
\text { transportation network }\end{array}$ & 0 & 0 & 0 & 0 & 0 \\
\hline \multirow[t]{10}{*}{ Investment } & Cabotage & 0 & 0 & 0 & 0 & 0 \\
\hline & Short sea shipping & 1 & 0 & 0 & 0 & 1 \\
\hline & Internal navigation & 0 & 0 & 0 & 0 & 0 \\
\hline & Internal shipping & 0 & 0 & 0 & 0 & 0 \\
\hline & Domestic shipping & 0 & 0 & 0 & 0 & 0 \\
\hline & Coastal shipping & 0 & 0 & 0 & 0 & 0 \\
\hline & "Transport cost" & 0 & 0 & 0 & 0 & 0 \\
\hline & Cabotage logistics & 0 & 0 & 0 & 0 & 0 \\
\hline & $\begin{array}{c}\text { Waterway } \\
\text { transportation network }\end{array}$ & 0 & 0 & 0 & 0 & 0 \\
\hline & Total & 4 & 4 & 0 & 0 & 8 \\
\hline
\end{tabular}

Fonte: Dados de pesquisa.

Frente ao exposto, a base de dados que apresentou maior representatividade foram as bases de dados Scopus e Web of Science, ambas com $50 \%$ das publicações do portfólio. As bases de dados IEEE e Science Direct não apresentaram aderência ao tema de pesquisa, sendo assim, não contribuiu com nenhum artigo no portfólio bibliográfico, apresentando $0 \%$ de representatividade, conforme é possível visualizar na Figura 3.

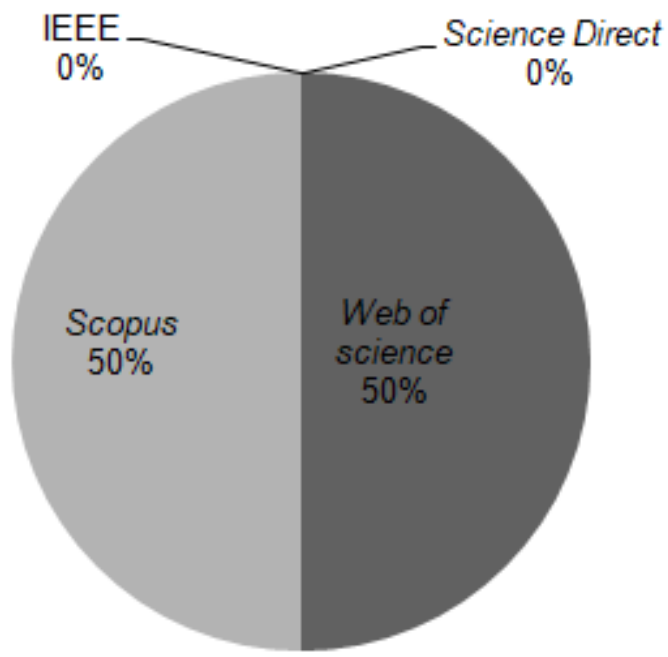

Figura 3 - Representatividade das bases de dados no portfólio bibliométrico. Fonte: Dados de pesquisa.

\subsection{Resultados}

Na presente seção será discutido os resultados da análise bibliométrica dos oito artigos presentes no portfólio. Dessa forma, será feito o estudo acerca da produção e autoria, conteúdo, citação dos artigos e estudo das referências bibliográficas.

\subsubsection{Estudo de produção e autoria.}


Neste tópico, o propósito foi analisar o número de publicações por ano, classificar os artigos de acordo com os autores e universidades que mais contribuíram acerca do tema de pesquisa e identificar os países que apresentaram o maior número de publicações.

Com o objetivo de avaliar a produção científica relacionada sobre o modal de transporte cabotagem e seus impactos na gestão portuária, construiu-se a Tabela 3 que demonstra o número de publicações dos artigos, contidos no portfólio, identificados por ano. Na coleta de dados não foi utilizada nenhuma restrição de tempo. Os artigos alinhados à pesquisa decorrem do ano de 2004 a 2018.

Tabela 3 - Número de publicações por ano

\begin{tabular}{c|c|c}
$\begin{array}{c}\text { Ano de } \\
\text { publicação }\end{array}$ & $\mathbf{N}^{\circ}$ de artigos & Representatividade \\
\hline 2004 & 1 & $12,5 \%$ \\
\hline 2007 & 1 & $12,5 \%$ \\
\hline 2009 & 1 & $12,5 \%$ \\
\hline 2012 & 1 & $12,5 \%$ \\
\hline 2013 & 1 & $12,5 \%$ \\
\hline 2016 & 1 & $12,5 \%$ \\
\hline 2017 & 1 & $12,5 \%$ \\
\hline 2018 & 1 & $12,5 \%$ \\
\hline Total & $\mathbf{8}$ & $\mathbf{1 0 0} \%$
\end{tabular}

$\mathrm{Na}$ análise dos países, obteve-se um total de sete países com publicações alinhadas à pesquisa. O país com maior participação foi a Croácia, com duas publicações, conforme exposto na Tabela 4.

Tabela 4 - Número de publicações por país

\begin{tabular}{c|c|c} 
País & $\mathbf{N}^{\circ}$ de artigos & Representatividade \\
\hline Croácia & 2 & $25 \%$ \\
\hline Brasil & 1 & $12,5 \%$ \\
\hline Espanha & 1 & $12,5 \%$ \\
\hline Grécia & 1 & $12,5 \%$ \\
\hline Inglaterra & 1 & $12,5 \%$ \\
\hline Nova Zelândia & 1 & $12,5 \%$ \\
\hline Rússia & 1 & $12,5 \%$ \\
\hline Total & $\mathbf{8}$ & $\mathbf{1 0 0} \%$
\end{tabular}

$\mathrm{Na}$ análise das universidades, obteve-se um número de oito universidades todas com uma publicação no portfólio sobre a temática. A lista das universidades está descrita na Tabela 5. 


\begin{tabular}{c|c|c} 
Universidade & $\begin{array}{c}\mathbf{N}^{\circ} \text { de } \\
\text { artigos }\end{array}$ & Representatividade \\
\hline Centre for Innovation in Transport & 1 & $12,5 \%$ \\
\hline Institute of Chartered Shipbrokers & 1 & $12,5 \%$ \\
\hline Universidade de Campinas & 1 & $12,5 \%$ \\
\hline University of Piraeus & 1 & $12,5 \%$ \\
\hline University of Split & 1 & $12,5 \%$ \\
\hline University of Zagreb & 1 & $12,5 \%$ \\
\hline Vladivostok State University of Economics and Service & 1 & $12,5 \%$ \\
\hline Total & $\mathbf{8}$ & $12,5 \%$ \\
\hline Fonte: Dados de pesquisa. & $\mathbf{1 0 0} \%$
\end{tabular}

Ao realizar a análise dos autores do portfólio, chegou-se no total de 19 autores. Utilizou o software Vosviewer ${ }^{\circledR}$ para realizar a interação entre os autores. Na Figura 4 percebe-se que não houve relação entre os autores dos artigos do portfólio.



Figura 4 - Autores presentes no portfólio bibliográfico.

Fonte: Dados de pesquisa.

\subsubsection{Análise de conteúdo}

Nesta etapa do estudo, analisou-se o conteúdo dos artigos com o propósito de verificar as palavras-chave que obtiveram uma maior frequência entre os artigos selecionados, número de autores por artigo, publicações por revistas, fator de impacto das revistas e áreas de conhecimento das revistas. 
$\mathrm{Na}$ análise de número de autores por artigo, do total do portfólio, $50 \%$ foi desenvolvido por quatro autores, $27,5 \%$ por três autores e $12,5 \%$ por um único autor, conforme demonstra a Tabela 6 .

Tabela 6 - Número de autores por artigo

\begin{tabular}{c|c|c}
$\mathbf{N}^{\circ}$ de autores & $\mathbf{N}^{\circ}$ de publicações & Representatividade \\
\hline 1 & 1 & $12,5 \%$ \\
\hline 2 & 3 & $27,5 \%$ \\
\hline 3 & 4 & $50 \%$ \\
\hline Total & $\mathbf{8}$ & $\mathbf{1 0 0 \%}$ \\
\multicolumn{2}{|c}{ Fonte: Dados de pesquisa. }
\end{tabular}

$\mathrm{Na}$ análise das palavras-chave, encontrou-se um total de 22 palavras presente em 3 artigos do portfólio, 5 artigos não apresentavam palavras-chave no documento. Desse modo, 12 palavras-chave foram mencionadas uma única vez e oito palavraschave apareceram pelo menos duas vezes nos artigos, sendo que Port e Sea foram as mais citadas, conforme a Tabela 7 .

Tabela 7 - Palavras-chave presentes no por
\begin{tabular}{c|c} 
Palavra-chave & Frequência \\
\hline Port & 4 \\
\hline Sea & 3 \\
\hline Competitivenes & 2 \\
\hline Development & 2 \\
\hline Management & 2 \\
\hline Transport & 2 \\
\hline Shipping & 2 \\
\hline System & 2 \\
\hline Short & 2 \\
\hline Cargo & 2 \\
\hline Font: Dados
\end{tabular}

Fonte: Dados de pesquisa.

$\mathrm{Na}$ análise das revistas dos artigos estudados, é demonstrado o número das que tiveram mais publicações acerca do tema de pesquisa. A revista Maritime Policy \& Management apresentou maior representatividade com três artigos publicados, representando $37,5 \%$ do portfólio, conforme a Tabela 8 .

Tabela 8 - Número de artigos por revista/jornal

\begin{tabular}{c|c|c} 
Revista & $\mathbf{N}^{\mathbf{0}}$ de artigos & Representatividade \\
\hline Maritime Policy \& Management & 3 & $37,5 \%$ \\
\hline Marine Intellectual Technologies & 1 & $12,5 \%$ \\
\hline Nase More & 1 & $12,5 \%$ \\
\hline Promet - Traffic \& Transportation & 1 & $12,5 \%$ \\
\hline Revista Espacios & 1 & $12,5 \%$ \\
\hline Transport Reviews & 1 & $12,5 \%$ \\
\hline Total & $\mathbf{8}$ & $\mathbf{1 0 0} \%$
\end{tabular}


Fonte: Dados de pesquisa.

Tendo em vista o estudo das revistas que estão indexados os artigos do portfólio, realizou-se a análise quanto ao impacto pelo SCImago Journal Rank (SJR) e o índice de Hirsch (índice $\mathrm{H}$ ). Estes índices visam classificar o desempenho e impacto de revistas científicas. O SCImago Journal \& Country Rank (SJR), utiliza o índice bibliográfico Scopus, lançado em dezembro de 2007. O SJR é resultado de um projeto conjunto entre o grupo SCImago e a Elsevier Publishing Co.

A revista Transport Reviews apresentou maior relevância, estando presente no primeiro quartil (Q1), score de 2,14 e índice $\mathrm{H}$ de 65. A revista Marine Intellectual Technologies não apresentou dados de SJR. Na Tabela 9 está representada a revistas, o respectivo quartil de impacto, score e índice $\mathrm{H}$.

Tabela 9 - Índice SJR das revistas

\begin{tabular}{c|c|c|c} 
Revista & Quartil & Score & H \\
\hline Transport Reviews & Q1 & 2,14 & 65 \\
\hline Maritime Policy \& Management & Q1 & 1.1 & 48 \\
\hline Promet - Traffic \& Transportation & Q2 & 0,27 & 15 \\
\hline Nase More & Q2 & 0,23 & 10 \\
\hline Revista Espacios & Q3 & 0,16 & 12 \\
\hline
\end{tabular}

Fonte: Dados de pesquisa.

No que tange a análise das áreas de conhecimento das revistas, fez-se a partir das áreas indexadas no SCImago Journal Rank. Observou-se 11 áreas distribuídas em cinco revistas analisadas. As áreas de Ciências Sociais e Engenharia estão presentes em três revistas, respectivamente, com representação de $60 \%$ nas revistas do portfólio. Na Tabela 10 estão representadas as áreas de conhecimento das revistas, bem como o número de revistas com determinada área e representatividade.

Tabela 10 - Áreas de conhecimento das revistas

\begin{tabular}{c|c|c} 
Área de conhecimento & $\mathbf{N}^{\circ}$ de artigos & Representatividade \\
\hline Ciências Sociais & 3 & $60,0 \%$ \\
\hline Engenharia & 3 & $60,0 \%$ \\
\hline Ciências Ambientais & 2 & $40,0 \%$ \\
\hline $\begin{array}{c}\text { Administração, Gestão e } \\
\text { Contabilidade }\end{array}$ & 1 & $20,0 \%$ \\
\hline Ciências da Decisão & 1 & $20,0 \%$ \\
\hline Engenharia Química & 1 & $20,0 \%$ \\
\hline
\end{tabular}

Fonte: Dados de pesquisa.

\subsubsection{Análise da citação dos artigos}

Uma das análises realizadas nos artigos que compõem o portfólio foi a citação por artigos, que consiste no número de vezes que cada artigo foi citado por outros autores. Com o auxílio do Google Acadêmico, no mês de Agosto de 2019, foi possível verificar que o artigo que mais foi citado em publicações foi o Logistics strategies for short sea shipping operating as part of multimodal transport chains, com 39 aparições, 
VI CIDESPORT/2019

Congresso Internacional

de Desempenho Portuário

seguido do artigo Competitiveness between short sea shipping and road freight transport in mainland port connections; the case of two Greek ports, que teve 23 citações, conforme a Tabela 11.

Tabela 11 - Número de citações por artigo

\begin{tabular}{c|c} 
Título do artigo & Citações \\
\hline $\begin{array}{c}\text { Logistics strategies for short sea shipping operating as part of multimodal transport } \\
\text { chains }\end{array}$ & 39 \\
\hline $\begin{array}{c}\text { Competitiveness between short sea shipping and road freight transport in mainland port } \\
\text { connections; the case of two Greek ports }\end{array}$ & 23 \\
\hline A qualitative analysis of reintroducing cabotage onto New Zealand's coasts & 18 \\
\hline Short Sea Shipping in Supply Chains. A Strategic Assessment & 10 \\
\hline Short Sea Shipping - an Opportunity for Development of the North Port of Split & 0 \\
\hline Management and prospects of the Croatian short sea shipping development & 0 \\
\hline Factors of competitiveness of coastal sea freight shipping & 0 \\
\hline Cabotage services in Brazil: Key benefits and current challenges & 0 \\
\hline
\end{tabular}

Fonte: Dados de pesquisa.

\subsubsection{Estudo das referências bibliográficas}

$\mathrm{Na}$ etapa de análise das referências bibliográficas, foram verificadas 202 citações, utilizadas nos oito artigos do portfólio. Na Tabela 12, pode-se observar que a citação mais utilizada nas publicações foi The Development of SSS in Europe. Seguida por Dynamic Alternative in Sustainable Transport Chain, da Commission of the European Communities, presente em quatro artigos do portfólio. E por fim, a referência Strengths and weaknesses of short sea shipping dos autores Paixão e Marlow, foi a segunda mais utilizada, citada em três artigos. Outros seis artigos foram citados duas vezes, sendo essa a menor quantidade de repetição de citações.

Tabela 12 - Referências usadas nos artigos do Portfólio

\begin{tabular}{c|c|c} 
Autores & Título & Citações \\
\hline $\begin{array}{c}\text { Commission of the } \\
\text { European Communities }\end{array}$ & $\begin{array}{c}\text { The Development of SSS in Europe. A Dynamic Alternative } \\
\text { in Sustainable Transport Chain. A Second Two Yearly } \\
\text { Progress Report, COM (99) 0317 Final }\end{array}$ & 4 \\
\hline $\begin{array}{c}\text { PAIXAO, A. C. e } \\
\text { MARLOW, P. B. }\end{array}$ & Strengths and weaknesses of short sea shipping & 3 \\
\hline Baird, A. J. & The economics of motorways of the sea & 2 \\
\hline $\begin{array}{c}\text { PAIXAO, A. C. e } \\
\text { MARLOW, P. B. }\end{array}$ & $\begin{array}{c}\text { The competitiveness of short sea shipping in multimodal } \\
\text { logistics supply chains: service attributes }\end{array}$ & 2 \\
\hline $\begin{array}{c}\text { PERAKIS, N. A. and } \\
\text { DENISIS, Ath. }\end{array}$ & A survey of short sea shipping and its prospects in the USA & 2 \\
\hline $\begin{array}{c}\text { Republic of Croatia, } \\
\text { Ministry of Sea, Tourism, }\end{array}$ & $\begin{array}{c}\text { Pre-Accession Maritime Strategy of the Republic of } \\
\text { Croatia, Ministry of Sea, Tourism, Transportation and } \\
\text { Development }\end{array}$ & 2 \\
\hline
\end{tabular}




\begin{tabular}{c|c|c}
\hline $\begin{array}{c}\text { Transportation and } \\
\text { Development. }\end{array}$ & \\
\hline Maritime Transport III & $\begin{array}{c}\text { S. S. Marchan: AnAnalysis of the Potential of the Short Sea } \\
\text { Shipping due to its Cost Structure }\end{array}$ & 2 \\
\hline $\begin{array}{c}\text { White Paper- European } \\
\text { Transport Policy for 2010 }\end{array}$ & Time to decide, European Commission & 2 \\
\hline
\end{tabular}

Fonte: Dados de pesquisa.

\section{CONSIDERAÇÕES FINAIS}

Este estudo teve como objetivo avançar na temática de pesquisas sobre o modal de transporte cabotagem e seus impactos na gestão portuária, através da análise bibliográficas de artigos disponíveis nas bases de dados online. Assim, foi realizada uma análise bibliométrica dos artigos dentro da temática nas bases de dados IEEE, Science Direct, Scopus e Web of Science.

A partir da análise nas bases de dados com a inserção de 27 palavras-chave, obteve-se um portfólio bibliográfico de oito artigos alinhados à temática de pesquisa. Para obter êxito nesse objetivo, foi utilizada a técnica da bibliometria com coleta de dados e análise de resultados mediante estudo de produção e autoria, análise de conteúdo, de citação e das referências bibliográficas dos artigos selecionados.

Através dos resultados encontrados na análise bibliométrica foi observado que os anos de pesquisas variaram de 2004 e 2018, com apenas uma publicação por ano.

$\mathrm{Na}$ análise dos países, obteve-se um total de sete países com publicações alinhadas à pesquisa. O país com maior participação foi a Croácia, com duas publicações.

No que tange o estudo das universidades, obteve-se um número de oito universidades todas com uma publicação no portfólio sobre a temática.

Ao realizar a análise dos autores do portfólio, chegou-se no total de 19 autores. Contudo, notou-se que não houve relação entre os autores dos artigos do portfólio.

Tendo em vista a análise do número de autores por artigo, quatro artigos foram desenvolvidos por quatro autores, três artigos escritos por três autores e um artigo publicado por um único autor.

No estudo das palavras-chaves presentes nas publicações, observou-se que 12 palavras-chave foram mencionadas uma única vez e oito palavras-chave apareceram pelo menos duas vezes nos artigos, sendo Port e Sea as palavras-chave mais citadas.

Entre as revistas com maior produção científica sobre o tema de pesquisa, destacou-se a revista Maritime Policy \& Management com três publicações sobre a temática.

A revista Transport Reviews apresentou maior relevância, estando presente no primeiro quartil (Q1), score de 2,14 e índice $\mathrm{H}$ de 65. Contudo, a revista Marine Intellectual Technologies não apresentou dados de SJR.

No que tange a análise das áreas de conhecimento das revistas, observou-se 11 áreas distribuídas em cinco revistas analisadas. As áreas de Ciências Sociais e Engenharia estão presentes em três revistas, respectivamente, com representação de $60 \%$ nas revistas do portfólio.

Com o auxílio do Google Acadêmico, foi possível verificar que o artigo que mais foi citado em publicações foi o Logistics strategies for short sea shipping operating as part of multimodal transport chains, com 39 aparições, seguido do artigo Competitiveness between short sea shipping and road freight transport in mainland port connections; the case of two Greek ports, que obteve 23 citações. 
$\mathrm{Na}$ etapa de análise das referências bibliográficas, foram verificadas 202 citações, utilizadas nos oito artigos do portfólio. Pode-se observar que a citação mais utilizada nas publicações foi The Development of SSS in Europe. Seguida por Dynamic Alternative in Sustainable Transport Chain, da Commission of the European Communities, presente em quatro artigos do portfólio.

Frente as análises realizadas no estudo, pode-se notar a grande relevância do estudo, obtendo uma visão mundial sobre os impactos da cabotagem na gestão dos portos. Todavia, o estudo limitou-se a oito publicações sobre a temática, dessa forma, sugere-se para pesquisas futuras abranger um número maior de bases de dados, bem como inserir novas palavras-chave sobre o tema, a fim de suprir lacunas do estudo.

\section{REFERÊNCIAS}

ANTAQ - Agência Nacional de Transporte Aquaviários. Anuário Estatístico Aquaviário 2010. Disponível em: <http://web.antaq.gov.br/ANUARIO/>. Acesso em: 13 ago. 2019.

ANTAQ - Agência Nacional de Transporte Aquaviários. Anuário Estatístico Aquaviário 2011. Disponível em: <http://web.antaq.gov.br/ANUARIO/>. Acesso em: 13 ago. 2019.

ANTAQ - Agência Nacional de Transporte Aquaviários. Anuário Estatístico Aquaviário 2012. Disponível em: <http://web.antaq.gov.br/ANUARIO/>. Acesso em: 13 ago. 2019.

ANTAQ - Agência Nacional de Transporte Aquaviários. Anuário Estatístico Aquaviário 2013. Disponível em: <http://web.antaq.gov.br/ANUARIO/>. Acesso em: 13 ago. 2019.

ANTAQ - Agência Nacional de Transporte Aquaviários. Anuário Estatístico Aquaviário 2014. Disponível em: <http://web.antaq.gov.br/ANUARIO/>. Acesso em: 13 ago. 2019.

ANTAQ - Agência Nacional de Transporte Aquaviários. Anuário Estatístico Aquaviário 2015. Disponível em: <http://web.antaq.gov.br/ANUARIO/>. Acesso em: 13 ago. 2019.

ANTAQ - Agência Nacional de Transporte Aquaviários. Anuário Estatístico Aquaviário 2016. Disponível em: <http://web.antaq.gov.br/ANUARIO/>. Acesso em: 13 ago. 2019.

ANTAQ - Agência Nacional de Transporte Aquaviários. Anuário Estatístico Aquaviário 2017. Disponível em: <http://web.antaq.gov.br/ANUARIO/>. Acesso em: 13 ago. 2019.

ANTAQ. Anuário Estatístico Aquaviário 2018. Disponível em: <http://web.antaq.gov.br/ANUARIO/>. Acesso em: 13 ago. 2019.

ANTAQ - Agência Nacional de Transporte Aquaviários (a). 2019. ANTAQ e Abac debatem navegação de cabotagem. Disponível em: $<$ http://portal.antaq.gov.br/index.php/2019/03/15/antaq-e-abac-debatem-navegacaode-cabotagem/>. Acesso em: 6 ago. 2019.

ANTAQ - Agência Nacional de Transporte Aquaviários (b). 2019. Aumenta o transporte no longo curso e na cabotagem. Disponível em: < http://portal.antaq.gov.br/index.php/2019/02/18/aumenta-o-transporte-no-longocurso-e-na-cabotagem/ >. Acesso em: 6 ago. 2019. 
VI CIDESPORT/2019

Congresso Internacional

de Desempenho Portuário

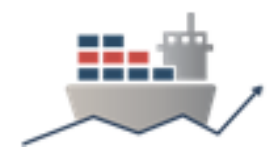

BARBOSA, L. P. Sustentabilidade e competitividade na cadeia logística: redução de emissões de gases de efeito estufa com o uso do modal de transporte cabotagem: estudo de caso. 2018. 78 p. Dissertação (Mestrado em Gestão para a Competitividade) - Escola de Administração de Empresas de São Paulo. Fundação Getulio Vargas. 2018.

BRASIL. Lei $\mathbf{n}^{\circ} \mathbf{9 . 4 3 2}$, de 8 de janeiro de 1997. Dispõe sobre a ordenação do transporte aquaviário e dá outras providências. Disponível em: <http://www.planalto.gov.br/ccivil_03/LEIS/L9432.htm>. Acesso em: 13 ago. 2019.

BENDALL, H. B.; BROOKS, M. R. Short sea shipping: Lessons for or from Australia? Annual Conference of The International Association of Maritime Economists: IAME, Lisboa, Julho, 2010.

EUROPEAN COMMISSION. The Development of Short Sea Shipping in Europe: A Dynamic Alternative in a Sustainable Transport Chain. Bruxelas, 1999. Disponível em: <https://www.vlaamsehavencommissie.be/sites/default/files/documenten/SSS001\%20EN.pdf>. Acesso em: 13 ago. 2019.

FLORENTINO, M. S. Gestão de custo no transporte marítimo de cargas no Brasil. 2010. 115 f. Dissertação (Mestrado) - Faculdade de Administração e Finanças, Universidade do Estado do Rio de Janeiro, Rio de Janeiro, 2010.

GIL, A. C. Como Elaborar Projetos De Pesquisa. 5 ed. São Paulo: Atlas, 2010.

MOURA, D. A. M.; BOTTER, R. C. O transporte por cabotagem no Brasil: potencialidade para a intermodalidade visando a melhoria do fluxo logístico. Revista Produção Online. Florianópolis, SC, v.11, n. 2, p. 595-617, abr./jun., 2011.

ROESCH, S. M. A. Projetos de estágio e de pesquisa em administração: guia para estágios, trabalhos de conclusão, dissertações e estudos de caso. $3^{a}$ ed. $-6^{a}$ reimp. - São Paulo: Atlas, 2015.

SEAFERS' RIGHTS INTERNACIONAL. Cabotage laws of the world. Londres, 2018. Disponível em: <http://ftp.elabor8.co.uk/sri/cabotage/flipbook/mobile/index.html>. Acesso em: 13 ago. 2019.

SILVA, A. E. Cabotagem versus transporte rodoviário: um estudo de caso da distribuição de arroz do sul catarinense para o nordeste brasileiro. 2014. 71 p. Monografia apresentada no Curso de Administração com Linha de Formação Especifica em Comércio Exterior da Universidade do Extremo Sul Catarinense UNESC para obtenção do grau de bacharel em Administração de Empresas.

SILVA, M. R.; HAYASHI, C. R. M.; HAYASHI, M. C. P. I. Análise bibliométrica e cientométrica: desafios para especialistas que atuam no campo. InCID: Revista de Ciência da Informação e Documentação. Ribeirão Preto, v. 2, n. 1, p. 110-129, jan./jun. 2011.

\section{APÊNDICE A}


VI CIDESPORT/2019

Congresso Internacional

de Desempenho Portuário

BUKLJAŠ, M.; JOLIĆ, N.; JOLIĆ, A. Management and Prospects of the Croatian

Short Sea Shipping Development. Promet -Traffic \& Transportation, v. 19, n. 2, p.

95-102, 2007.

CASACA, A. P.; MARLOW, P. B. Logistics strategies for short sea shipping operating as part of multimodal transport chains. Maritime Policy \& Management, v. 36, n. 1, p. 1-19, 2009.

CAVANA, R. Y. A qualitative analysis of reintroducing cabotage onto New Zealand's coasts. Maritime Policy \& Management, v. 31, n. 3, p. 179-198, 2004.

OLIVEIRA, A. R. de; SÁ PORTO, P. C. de. Serviços de cabotagem no Brasil: principais vantagens e desafios atuais. Revista ESPACIOS, v. 37, n. 8, p. 1-12, 2016.

ISAEV, A. A.; MEGEY, E. S.; LENTAREV, A. A. Factors of competitiveness of coastal sea freight shipping. Marine intellectual technologies, v. 3, n. 4, p. 144-150, 2017.

MORALES-FUSCO, P.; SAURÍ, S.; de M., G. Short sea shipping in supply chains. A strategic assessment. Transport Reviews, v. 33, n. 4, p. 476-496, 2013.

SAMBRACOS, E.; MANIATI, M. Competitiveness between short sea shipping and road freight transport in mainland port connections; the case of two Greek ports. Maritime Policy \& Management, v. 39, n. 3, p. 321-337, 2012.

VUKIĆ, L.; BOLJAT, H. U.; SLIŠKOVIĆ, M. Short Sea Shipping-an Opportunity for Development of the North Port of Split. NAŠE MORE, v. 65, n. 3, p. 18-25, 2018. 